\title{
THE HERTZSPRUNG-RUSSELL DIAGRAMS FOR OPEN CLUSTERS
}

\author{
K. A. BARKHATOVA \\ State University, Sverdlovsk, USSR
}

The most important relationship between physical characteristics of stars is the spectrumluminosity correlation. Investigating the character of this correlation for stars of open clusters, Trumpler has worked out a classification of clusters according to their spectral composition.

A comparison of HR diagrams for open clusters with the standard sequence of stars in the solar neighborhood reveals a number of peculiarities. Usually there are no yellow or red giants in clusters containing $\mathrm{O}$ and $\mathrm{B}$ stars, while in some cases supergiants of later classes may occur among them. The giant branch is found in those clusters whose main sequence begins with A stars, or with later type stars. It should be noted that the absolute magnitudes of the brightest stars in a cluster are different for various clusters, varying from $-7.5^{\mathrm{m}}$ to $+2^{\mathrm{m}}$.

One of the peculiarities of the cluster diagrams is the shift of the upper parts of main sequences to the right. Most clusters consist of stars of various spectral classes. This may be due to the fact that stars of a cluster either did not originate simultaneously and are of different age, or they appeared at the same time, but differed in their initial masses and the rate of their evolution along the main sequence. Study of the spectrum-luminosity and colour-luminosity correlations in clusters is of primary importance for the problem of stellar evolution since clusters are undoubtedly systems of stars of common origin. But the diagrams of clusters are of interest not only from the point of view of a possible interpretation of stellar evolution. From the spectrum-luminosity diagrams one can also estimate the distance to the clusters. Since the trigonometrical parallaxes of only the nearest clusters are known to us, this method is the best for the determination of the distances of clusters. Bearing these two aims in mind we have collected in the course of the last few years all the published data on the colour-magnitude diagrams of clusters [2-35] and compiled an atlas, which was distributed among the Symposium participants. It is true that the material collected by us is not homogeneous. Besides the diversity of photometric systems there are differences in the limiting magnitudes of stars and in the accuracy of determinations of stellar magnitudes and colour-indices.

Besides the diagrams obtained as a result of photoelectric measurements there are also diagrams for which the magnitudes of stars were determined photographically.

As the stellar magnitudes and the colour-indices of stars usually refer to different photometric systems they have been separately reduced to the international system. In a number of cases some diagrams based on different sources are given for the same cluster. We shall now dwell on the characteristics of the diagrams of some clusters.

When discussing HR diagrams of clusters main attention was usually paid to the deviation of the upper ends of the main sequences. Only quite recently, investigating stars in the field of the Orion nebula, P. P. Parenago [I3] paid attention to the quite unusual behaviour of the fainter end of the main sequence. Walker [36] found that the Russell diagram, for NGC 2264 and NGC 6530, consists of the normal main sequence, which stretches from $\mathrm{O}$ to Ao type stars, and of stars of much later spectral classes, situated higher than the main sequence, 
in the domain of subgiants. The shape of the diagrams for the above mentioned clusters somewhat recalls the diagrams for NGC 2437, NGC 2126, NGC 228I.

But the earliest stars of these clusters belong to the classes B9-Ao. On the HR diagram for the clusters NGC 228I and NGC 2126 there is something like a continuous transition from the upper bright main sequence stars into the giant branch. Thus the Hertzsprung gap is filled. If these stars are cluster members, their position on the diagram is unusual. In the recently published work by Rach and Vasilevskis [37] it is pointed out that the Hertzsprung gap evidently does not exist in the Russell diagram for NGC 6940 cluster.

Many clusters with stars of earlier spectral classes are connected with diffuse nebulae and the main sequence of these clusters deviates upwards and to the right. Thus, for instance, in some cases the upper ends are situated perfectly vertically covering the interval of four and

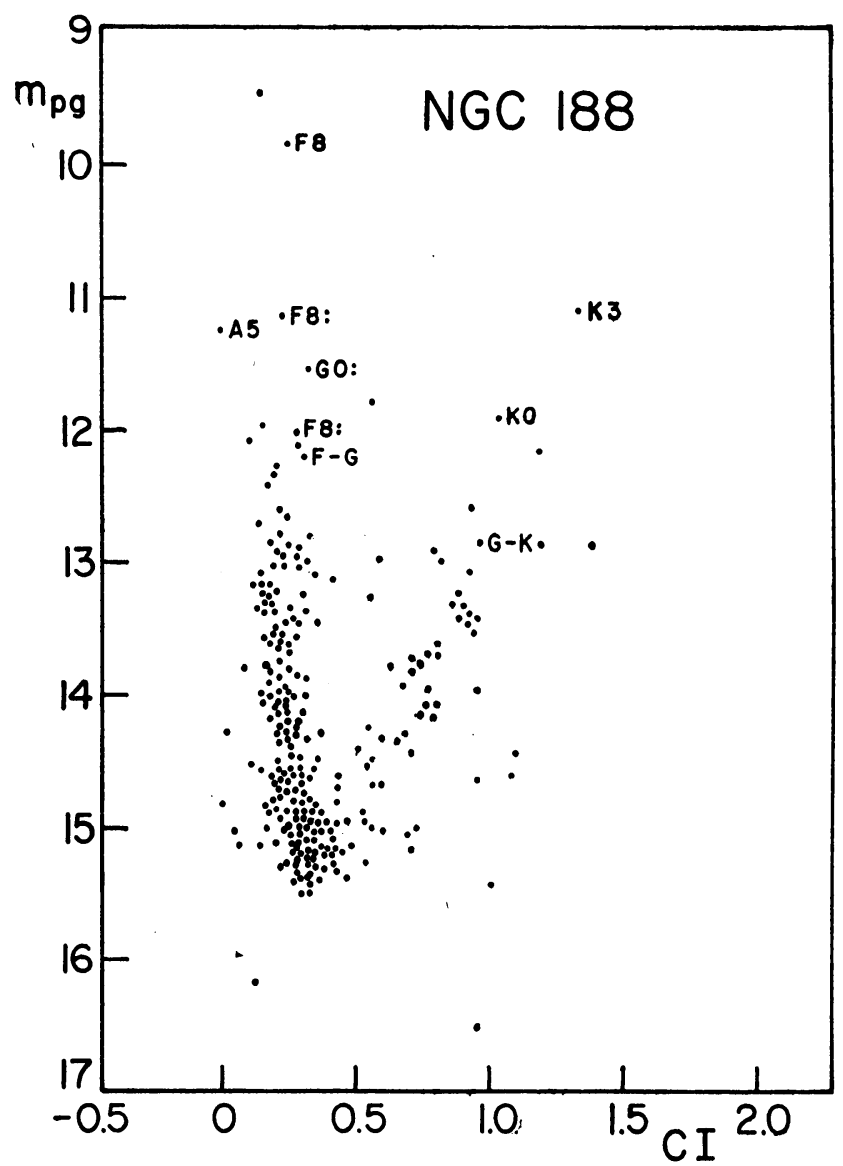

FIGURE I. A new HR diagram for the cluster NGC I88. more magnitudes (Orion, NGC 6823, AC I805, NGC I893). The upper end of the main sequence for NGC 2323 stretches upwards to $6^{\mathrm{m}}$, almost vertically. According to Münch's list [38] there are stars of high luminosity in the field of this cluster. The double cluster in Perseus contains along with hot giants, red supergiants of type $M$ and emission-line stars. Of great interest is the colour-magnitude diagram of the NGC 6830 cluster [25] situated at a distance of $2^{\circ}$ from NGC 6823, which is evidently a part of the stellar association in Vulpecula.

In the HR diagram of NGC 6830 the main sequence has the outlines characteristic for clusters containing high luminosity stars. There is a group of stars on the diagram situated in the region of bright giants. The shapes of the HR diagrams of clusters are very diverse, but the differences in the main sequences are most prominent when comparing the diagrams of such clusters as the Pleïades on the one hand, and Praesepe and the Hyades on the other.

The colour-magnitude diagrams of stars in Praesepe and the Hyades have a branch of giants besides the main sequence. The rich clusters NGC 6705, NGC 2099 belong to the group of clusters containing giants.

Much attention has been paid recently to the investigation of HR diagrams of NGC 752, NGC 2682, which have a number of peculiarities distinguishing them from other open clusters. The shape of the diagram for cluster NGC 2682 resembles the diagrams of globular clusters. 
As has been recently stated [27] the diagram for NGC I88, (Fig. I), is of an unusual shape similar to that of NGC 2682.

On the colour-magnitude diagram for stars of NGC I88 cluster one can clearly see not only the main sequence, but also its deviation off to the right and stretching into the region of yellow and red giants. Quite recently Bartaya of the Abastumani Observatory obtained a photograph of NGC I88 with an objective prism. P. P. Parenago has determined the spectra of stars. It was discovered that the cluster consists of stars of late spectral classes. Stars of $\mathrm{F}_{2}-\mathrm{F}_{5}$ type are the earliest and apparently there are also stars of later spectral classes. No considerable absorption of light has been discovered in the direction of the cluster. Its distance is I200 parsecs. NGC I88 and NGC 2682 are situated at considerable distances from the plane of the Galaxy. They have a considerable number of stars and seem to take an intermediate position between the globular and open clusters.
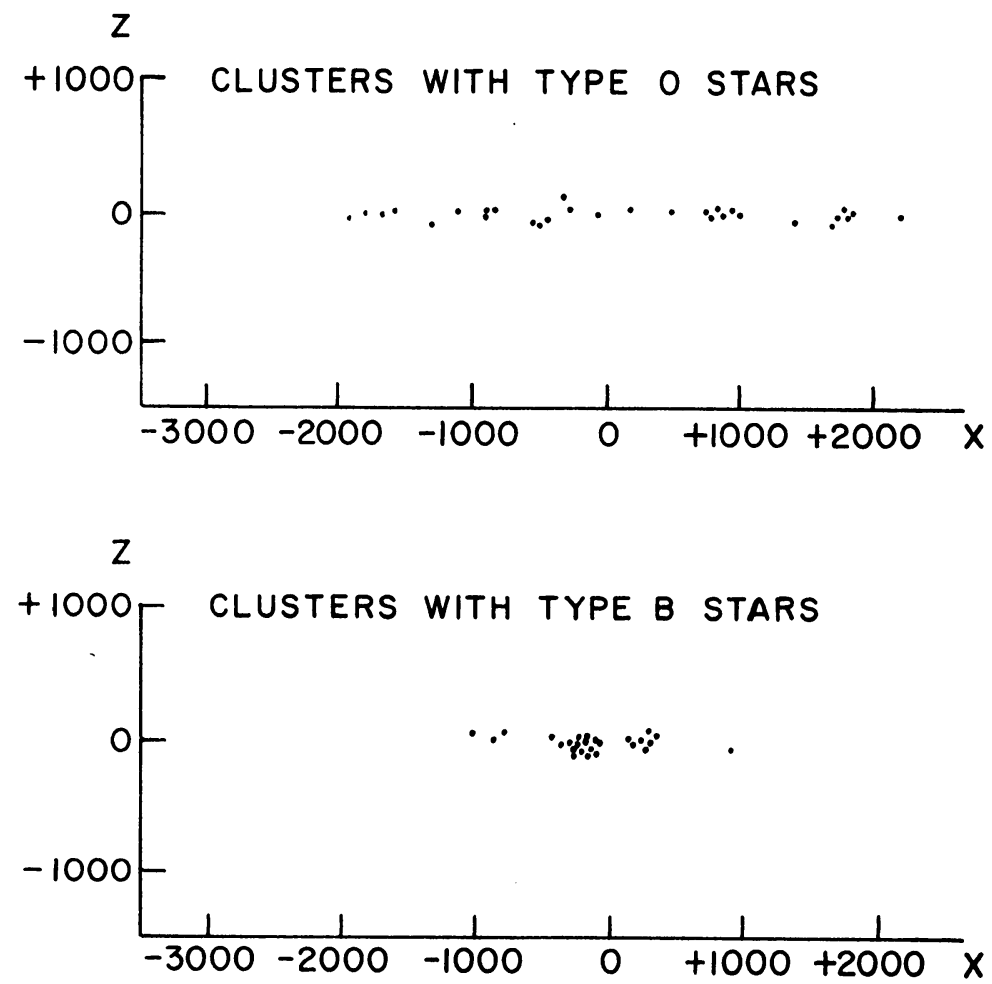

The type of the spectrum-luminosity diagram is a most important evolutionary characteristics of a cluster, especially if connected with the peculiarities of spatial distribution, motion and the structure of different types of clusters.

The following three groups of clusters have been chosen to compare the regularities in the spatial distribution of open clusters with their physical characteristics :

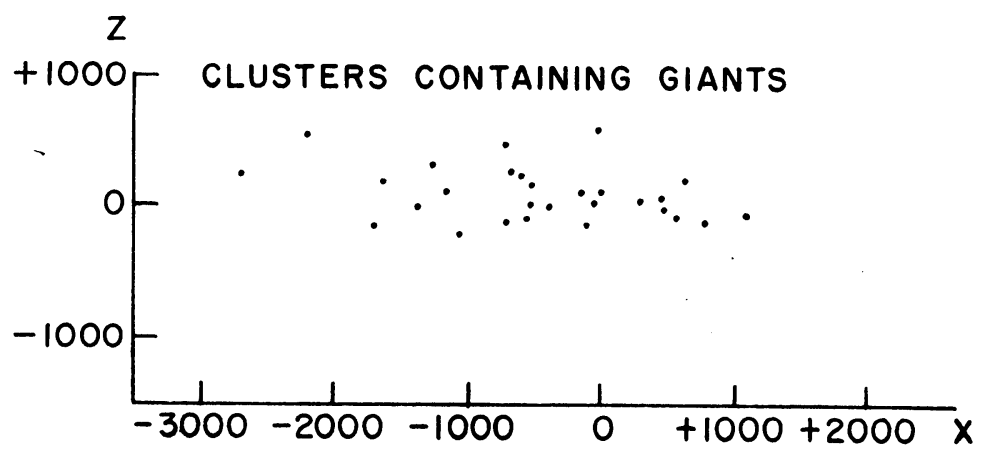

FIGURE 2. The distribution in the $(X, Z)$ coordinate plane of different types of clusters. The clusters with giants extend to greater heights from the galactic plane.

I. Clusters containing stars of earlier spectral class $\mathrm{O}$ or $\mathrm{B}$ which are often connected with diffuse nebulae;

2. Clusters containing only the main sequence stars beginning with class $\mathrm{Br}$;

3. Clusters containing, besides the main sequence stars, also giants. 
In Figure 2 which shows the distribution of these groups of clusters in the direction perpendicular to the plane of the Galaxy, the different spatial distribution corresponds to different types of clusters. This difference becomes particularly clear when we compare the first and the third group of clusters. The clusters connected with diffuse nebulae and containing stars of spectral classes $\mathrm{O}$ or Bo form a flatter subsystem. The clusters of this type are observed at considerable distances from the sun and have a small $Z$-coordinate $(Z=33 \mathrm{ps})$.

The clusters which include yellow and red giants have a much smaller concentration towards the plane of the Galaxy. These clusters form an intermediate subsystem and are at a distance of approximately $Z=\mathrm{I}_{50} \mathrm{ps}$.

\section{REFERENCES}

[I] R. Trumpler P. A. S. P. 37, 307, 1925.

[2] R. Zug, Lick Obs. Bull. 16, No. 454, II9, 1935.

[3] W. Becker, Zs. f. Ap. 30, 164, 1952.

[4] W. Becker u. J. Stock, Zs. f. Ap. 34, I, 1954 .

[5] H. Johnson, Ap. J. 117, 356, I953; 116, 649, I952; 119, I81, 1954; 119, I 85, 1954.

[6] H. Johnson, W. Morgan, Ap. J. 114, 522, 1951; 117, 313, 1953; 119, 344, 1954; 122, 429, 1955.

[7] A. N. Cox, Ap. J. 119, I88, I954; 121, N 3, I955.

[8] H. Weaver, Ap. J. 116, 612, 1952; 117, 366, I 953.

[9] M. Walker, Astr. J. 62, No. 1245, 37, 1957; Ap. J. 125, 636, I957, Suppl. 2, 365 (No. 23), I 956.

[Io] A. Hogg, G. Kron, Astr. J. 60, No. I233, I955; M. N. 113, 746, I953.

[II] J. Cuffey, Harv. Ann. 105, 403, I937; 106, I39, I938; Ap. J. 123, 59, 1956.

[12] E. Boden, Uppsala Ann. 2, No. I, 1946, 2, No. 7, 1950, 3, No. 4, 195 I.

[13] P. P. Parenago, Sternb. Publ. 25, 1954.

[14] L. Binnendijk, Leiden Ann. 19, 2, 1947.

[15] S. Sharpless, Ap. J. 119, I, I954.

[16] A. Wallenquist, Uppsala Medd No. 65, 1936; Ann v.d. Bosscha 3, 3, 1931; 5, 3, I932.

[17] H. Zeipel, J. Lindgren, Kungl. Svensk. Veten. Handl. B 6I, No. or 5, I92 Í.

[18] G. Larsson-Leander, Stockh. Ann. 20, No. 2, 1957.

[19] A. van Maanen, Ap. J. 100, I, 1944.

[20] E. Ebbighausen, Astr. J. 90, No. 6, I939.

[21] N. B. Grigorieva, Astr. Circ. No. I78, 21, 1957.

[22] N. Roman, Ap. J. 121, No. 3, I953.

[23] N. Roman, W. Morgan, Ap. J. 111, 426, 1950.

[24] D. Harris, W. Morgan, N. Roman, Ap. J. 119, 622, 1954.

[25] K. A. Barkhatova, A. Zh. 33, 4, 5, 6, 1956; 34, 2, 6, I957.

[26] K. A. Barkhatova, Scientific papers of the Ural A. M. Gorky State University, No. 22, 1958.

[27] K. A. Barkhatova, New data on the diffuse stellar cluster NGC I88, Astr. Circ. No. I9I, 1958.

[28] K. A. Barkhatova, L. I. Driakhlushina, A. Zh. USSR 35, 3, $195^{8}$.

[29] W. Becker, E. Müller, U. Steinlin, Zs. f. Ap. 38, 2, I955.

[30] G. Miczaika, Astr. J. 59, No. 7, I955.

[3I] J. Cuffey, Ap. J. 94, 55, r94I.

[32] J. Cuffey, Ap. J. 97, 93, I943.

[33] H. Johnson, C. Knuckles, Ap. J. 122, 209, I955.

[34] H. Johnson, A. Sandage, Ap. J. 121, 6I6, I955.

[35] H. Johnson, W. Hiltner, Ap. J. 123, 267, 1956.

[36] M. Walker, Nonstable stars, Erevan 1957, p. $4^{2 .}$

[37] Vasilevskis, Rach, Astr. J. 62, No. 6, I75, I957.

[38] G. Münch, Ap. J. 113, No. 2, r95r. 